\title{
Role of Pre and Post Cytocentrifugation and Different Media in the Primary Isolation of Fungi
}

\author{
Anuja Sharma ${ }^{1, *}$, Ravinder Kaur ${ }^{2}$ \\ ${ }^{1}$ Department of Microbiology, Kurukshetra University, India \\ ${ }^{2}$ Department of Microbiology, Maulana Azad Medical College, New Delhi, India
}

Copyright $@ 2017$ by authors, all rights reserved. Authors agree that this article remains permanently open access under the terms of the Creative Commons Attribution License 4.0 International License

\begin{abstract}
The present study was undertaken to compare the role of direct microscopy with microscopy after cytocentrifugation during diagnosis of fungal infections. Also, the role and usefulness of different media like Sabouraud Dextrose Agar (SDA), Emmons Sabouraud Dextrose Agar (ESDA) and Inhibitory Mold Agar (IMA) for the growth of different fungi were compared to determine the most effective primary medium to be used in routine. Out of the total number of observations, $11.25 \%$ showed 2 folds increase, $28.75 \%$ showed 3 folds increase, 20\% showed 4 folds increase and $40 \%$ showed 5 folds or more increase in cell density after cytocentrifugation. Also there were 3.75\% of negative observations during direct microscopy while positive results were obtained on microscopy of same samples after cytocentrifugation. On studying the effect of various primary media on isolation and growth of different fungi, a total of 27 fungal isolates were recovered on culture media and identified. These isolates represented six important genera of fungi namely Candida, Penicillium, Trichophyton, Aspergillus, Cryptocococcus and Rhizopus. Out of a total of 27 fungal isolates, $66.66 \%$ fungal isolates were isolated on all the three media, $11.11 \%$ on SDA alone, $7.40 \%$ on Emmons SDA alone and $14.81 \%$ on IMA alone.
\end{abstract}

Keywords Cytocentrifugation, Emmons Sabouraud Dextrose Agar, Inhibitory Mold Agar, Microscopy, Sabouraud Dextrose Agar

\section{Introduction}

Medical mycology has become an extremely challenging field of due to the infections caused by a wide and taxonomically diverse array of opportunistic fungi. Most of the fungi isolated from clinical samples are pathogenic and can cause infection in a sufficiently immunocompromised host and thus, should not be discarded as a contaminant [1, 2]. There has been a significant increase in the frequency of invasive mycoses due to opportunistic fungal pathogens and the emergence of less common but medically important fungal pathogens, especially in the increasing population of immunocompromised patients. Opportunistic mycoses pose diagnostic and therapeutic challenges and diagnosis of these fungal infections includes both the clinical suspicion and the retrieval of appropriate clinical specimen for microscopy, culture and histopathology. However, isolation and identification of the infecting organisms is extremely important for the proper diagnosis and management of infections due to not so common opportunistic fungi $[1,2]$.

Identification of any microorganism from a given sample involves two major steps: Microscopy and Culture. Microscopy includes the direct examination of sample either by direct wet mount or staining depending on the sample. Microscopy of samples like wet mount of urine sample, $\mathrm{KOH}$ wet mount of nail clipping, gram staining and India ink of CSF plays an important role in identification of microorganisms. Increasing frequency of fungal infections and importance of the role of microscopy in diagnosis has necessitated the usage of cytocentrifuge in many laboratories. The Cytospin system uses both the principles of centrifugation and fluid absorption to allow the deposition of a thin layer of cells on glass slide in the form of round or rectangular areas. Earlier it was used mainly for cerebrospinal fluid, but now cytocentrifugation is thought to be useful for several body fluids including urine, sputum, ascites and cerebrospinal fluid $[3,4]$

Although microscopy plays an important role in the diagnosis but it is important that in order to correctly diagnose and treat these infections the etiologic agent be grown and isolated on culture, and the organism be identified to ensure the proper treatment. It is therefore essential that an effective media, selective for fungi be utilized for all fungal cultures [5-7]. Different primary media used routinely for the isolation of fungi are one medium containing no antimicrobial agents, one medium containing an antibiotic(s) (commonly chloramphenicol and/or gentamicin) to prevent the growth of bacteria, one medium containing an antibiotic and additionally cycloheximide to inhibit the growth of rapidly growing saprophytic fungi [7-9]. 
Sabouraud dextrose agar (SDA), without any antibiotic is widely used as the standard medium for the primary isolation of fungal strains. Emmons Sabouraud dextrose agar, a modification of Sabouraud dextrose agar (SDA) has low $\mathrm{pH}$ of approximately 5.6 which is favorable for the growth of dermatophytes, while inhibiting contaminants [7]. Inhibitory mold agar (IMA) contains chloramphenicol (with some formulations containing Gentamicin) and supports the growth of a wide range of fungi and inhibits bacterial growth more effectively due to its antibiotic content [6, 7]. Hence this study was undertaken to compare direct microscopy with microscopy after cytocentrifugation and to compare the role and usefulness of different media like SDA, Emmons SDA and IMA for the growth of different fungi and determine the most effective primary medium to be used in routine.

\section{Methodology}

Clinical specimens submitted for fungal microscopy and culture in the Mycology laboratory, Department of Microbiology, Maulana Azad Medical College, New Delhi, over a period of three months were included in this study. Specimens from various sites (respiratory, urine, genital, skin, nails, tissue, CSF, other body fluids) were subjected to both microscopy and culture. A portion of the specimen was used for smear preparation by cytocentrifugation. One milliliter of sample was transferred to an assembled 1-ml disposable Cyto-Tek Centrifuge tube attached to a slide chamber containing disposable glass slide. This slide chamber was placed in the cytocentrifuge (Sakura Finetek, India) and centrifuged at 2,500rpm for 10 mins as specified by the manufacturer. Both pre and post cytocentrifugation microscopy was done, observations were made, and differences in the observations were noted, tabulated and analyzed. Two sets with one tube each of SDA, Emmons SDA and IMA were inoculated with the sample. One set was incubated at $22^{\circ} \mathrm{C}$ and the other set was incubated at $37^{\circ} \mathrm{C}$.
Tubes were inspected every alternate day for the presence of any growth up to 4-6 weeks. Fungal colonies on the media were semi quantitatively assessed and recorded. All isolates were subsequently identified and categorized according to the type of fungus grown and the media on which the growth was observed.

\section{Results}

Out of a total of 69 samples, $52.17 \%$ yielded a fungal etiology with $13.04 \%$ by microscopy alone, $21.73 \%$ by culture alone and $17.39 \%$ by both culture and microscopy. Out of the total number of 80 observations on microscopy, $11.25 \%$ showed 2 folds increase, $28.75 \%$ showed 3 folds increase, $20 \%$ showed 4 folds increase and $40 \%$ showed 5 folds or more increase in cell density after cytocentrifugation.(table:1) 5 folds or more increase in cell density was seen in maximum number of observations(32 out of 80 ). Also there were $3.75 \%$ of negative observations on direct microscopy but showed positive results after cytocentrifugation.

A total of 27 fungal isolates were recovered on culture and identified representing six important genera of fungi. The fungal isolates comprised of Candida albicans (37\%), Non albicans candida sp. (22\%)., Penicillium sp. (11\%), Trichophyton sp. (7\%), Aspergillus flavus (7\%), Aspergillus fumigatus (4\%), Aspergillus niger (4\%), Cryptococcus sp. (4\%), and Rhizopus sp. (4\%). Candida sp. (59\%) were the most common fungus isolated, with $37 \%$ Candida albicans and $22 \%$ Non albicans candida sp. Out of a total of 27 fungal isolates, $66.66 \%$ fungal isolates were isolated on all the three media, $11.11 \%$ on SDA alone, $7.40 \%$ on Emmons SDA alone and $14.81 \%$ on IMA alone (Fig. 1). Candida sp. and Rhizopus sp. were seen to grow on all the three media while Trichophyton sp. was only isolated from Emmons SDA and IMA (Table- 2).

Table 1. Microscopy profile postcytocentrifugation

\begin{tabular}{|c|c|c|c|c|c|}
\hline \multirow{2}{*}{ Sr. No. } & \multirow{2}{*}{$\begin{array}{c}\text { Increase in cell } \\
\text { density }\end{array}$} & \multicolumn{4}{|c|}{ No. of Observations (pus cells, budding yeast cells, fungal hyphae) $(\mathrm{n}=80)$} \\
\cline { 3 - 6 } & & Wet Mount (\%) & Gram Stain (\%) & India ink (\%) & Total (\%) \\
\hline 1. & 2 folds & $00(0)$ & $07(8.75)$ & $02(2.5)$ & $09(11.25)$ \\
\hline 2. & 3 folds & $04(5)$ & $15(18.75)$ & $04(5)$ & $23(28.75)$ \\
\hline 3. & 4 folds & $00(0)$ & $12(15)$ & $04(5)$ & $16(20)$ \\
\hline 4. & 5 folds or more & $03(3.75)$ & $20(25)$ & $09(11.25)$ & $32(40)$ \\
\hline
\end{tabular}


Table 2. Culture isolations on different media

\begin{tabular}{|c|c|c|c|c|c|c|}
\hline \multirow{2}{*}{ Sr. no. } & \multirow{2}{*}{ Fungal isolates } & \multirow{2}{*}{$\%$ of isolates } & \multicolumn{4}{|c|}{$\%$ of isolates } \\
\hline & & & On all the three media & Only on SDA & Only on Emmons SDA & Only on IMA \\
\hline 1. & Candida albicans & 37 & 100 & 0 & 0 & 0 \\
\hline 2. & Non albicans candida & 22 & 100 & 0 & 0 & 0 \\
\hline 3. & Cryptococcus spp. & 4 & 100 & 0 & 0 & 0 \\
\hline 4. & Trichophyton spp. & 7 & 0 & 0 & 50 & 50 \\
\hline 5. & Aspergillus flavus & 7 & 0 & 50 & 0 & 50 \\
\hline 6. & Aspergillus fumigatus & 4 & 0 & 0 & 100 & 0 \\
\hline 7. & Aspergillus niger & 4 & 0 & 0 & 0 & 100 \\
\hline 8. & Penicillium spp. & 11 & 0 & 66.66 & 0 & 33.34 \\
\hline 9. & Rhizopus spp. & 4 & 100 & 0 & 0 & 0 \\
\hline 10. & Total & 100 & 66.66 & 11.11 & 7.40 & 14.81 \\
\hline
\end{tabular}

Table 3. Comparison of primary growth on different media

\begin{tabular}{|c|c|c|c|c|c|c|}
\hline \multirow{2}{*}{$\begin{array}{c}\text { Sr. } \\
\text { no. }\end{array}$} & \multirow{2}{*}{ Category of fungus } & \multirow{2}{*}{$\begin{array}{c}\text { \% of } \\
\text { isolates }\end{array}$} & On all the three media & Only on SDA & Only on Emmons SDA & Only on IMA \\
\cline { 4 - 6 } & & 62.96 & 100 & 0 & 0 \\
\hline 1. & Yeast & 25.92 & 7.4 & 0 & 42.85 & 0 \\
\hline 2. & Hyaline hyphomycetes & 3.7 & 100 & 0 & 5.28 \\
\hline 3. & Dermatophytes & 100 & 66.66 & 11.11 & 0 \\
\hline 4. & Zygomycetes & Total & & & 0 \\
\hline 5. & & & & & 0 \\
\hline
\end{tabular}

\section{Discussion}

An overall $77.77 \%$ of the isolates were recovered on SDA, $74.06 \%$ of the isolates were recovered on Emmons SDA and $81.47 \%$ were recovered on IMA. When considering isolates that grew only on one media, IMA supported the growth of 2 times more isolates than did Emmons SDA. There was no difference in the time of recovery of the isolates on all the three media. Yeasts including Candida and Cryptococcus grew on all the three media (Table: 3). Dermatophytes showed no recovery on SDA and grew only on Emmons SDA and IMA (Table: 3). None of the fungal isolates showed significant recovery on SDA over IMA which has also been found in another study conducted by Scognamiglio and coworkers at New York-Presbyterian Hospital, New York where overall, 94.2\% of the isolates recovered on IMA, with $75.1 \%$ of the isolates recovered on SDA, IMA supporting the growth of four times more isolates than did SDA [7]. However, in our study there are a few numbers of isolates (A. flavus, A. fumigatus, Trichophyton sp.) of certain genera (including hyaline hyphomycetes and dermatophytes) that grew on SDA and not IMA. (Table: 2 and 3).

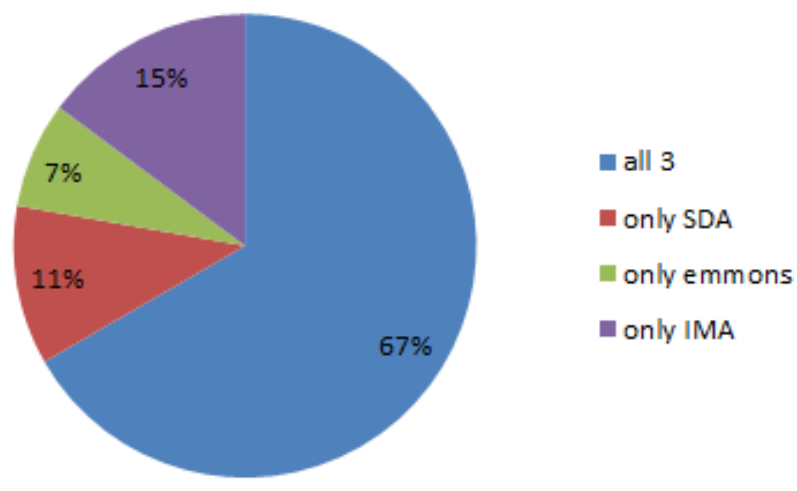

Figure 1. Fungal isolates on different media

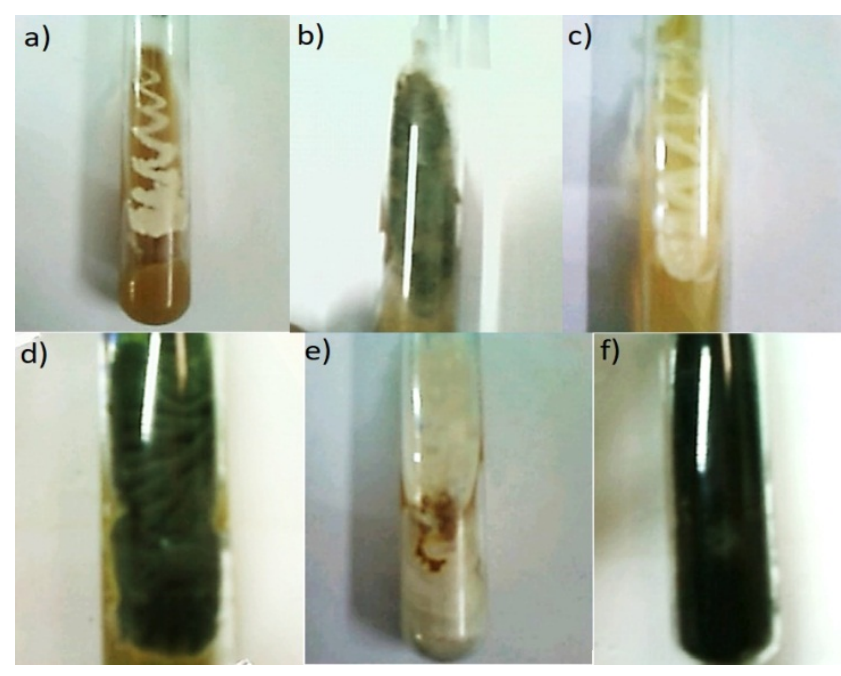

Figure 2. a) Candida sp.; b) Aspergillus flavus; c) Cryptococcus sp.; d) Peniciilium sp.; e) Trichophyton sp.; f) Rhizopus sp.

Our study supports that microscopy should be combined with cytocentrifugation to increase the standard of diagnosis and cytocentrifugation should be used for all kinds of body fluids including urine, sputum, ascites and spinal fluid and not only for CSF to avoid significant increase in errors during direct microscopy. Also it was seen that cytocentrifugation has advantages like decreased screening time, higher number and concentration of cells with better staining quality despite its longer preparation time. Studies have been conducted comparing cytospin technique with conventional smear preparation method and diverse results are available. A study conducted in France found cytocentrifugation using disposable chambers to give excellent results if one considers cellularity, fixation and the comfort for screening and also found it to be cost efficient when compared to other techniques like Cytyc Thinprep liquid-based cytology technique [4]. Another study done in 
Department of Pathology, University of Texas, Medical branch on sputum specimens for the detection of AFB discusses the added expense of cytocentrifugation for routine concentration of sputum specimens for the preparation of smears for detection of AFB and found cytocentrifugation to be useful when immediate preparation of a smear for the detection of AFB is requested, especially when the equipment is already available [11]. Also, another study conducted by Saceanu and coworkers concluded the presence of consistently higher number of cells on Cyto-Tek positive smears compared to parallel smears made by the traditional smear preparation technique [12]. In yet another study, 98 randomly taken samples of serous effusions were subjected to microscopy using conventional smear and cytospin preparation. Out of 24 malignant effusions only 14 were diagnosed on conventional smear and 23 in cytocentrifuge smear. Thus using cytocentrifuge smear diagnostic yield increased by 34.2\% [13].

In comparison of IMA and SDA as the routine medium for the primary isolation of fungi, IMA is superior to SDA as it recovered more isolates than did SDA. However SDA continues to have an important role in laboratory as some isolates were recovered only on SDA. This study also indicates that SDA should be used along with IMA or another proven supportive fungal medium containing antibiotics to avoid bacterial contamination. Emmons SDA can only be employed as an additional media for the better recovery of dermatophytes in laboratory.

\section{Conclusions}

This study was undertaken to compare direct microscopy with microscopy after cytocentrifugation and to compare the role of different media like Sabouraud dextrose agar (SDA), Emmons Sabouraud dextrose agar (ESDA) and Inhibitory mold agar (IMA) for the growth of different fungi and determine the most effective primary medium to be used in routine. Our study suggested that microscopy should be combined with cytocentrifugation to increase the standard of diagnosis and cytocentrifugation should be used for all kinds of body fluids including urine, sputum, ascites and spinal fluid and not only for CSF to avoid significant increase in errors during direct microscopy. It was also found that IMA is superior to SDA as it recovered more isolates than did SDA. However SDA continues to have an important role in laboratory as some isolates were recovered only on SDA. This study also indicates that SDA should be used along with IMA or another proven supportive fungal medium containing antibiotics to avoid bacterial contamination.

\section{Conflict of Interests}

The authors declare that there is no conflict of interests regarding the publication of this paper.

\section{REFERENCES}

[1] T.J. Walsh, A. Groll, J. Hiemenz, R. Flemming, E. Roilides, and E. Anaissie. Infections due to emerging and uncommon medically important fungal pathogens, Journal of Clinical Microbiology, Vol. 1, pp. 48-66, 2004.

[2] M.A. Pfaller and D.J. Diekema. Rare and Emerging Opportunistic Fungal Pathogens: Concern for Resistance beyold Candida albicans and Aspergillus fumigates, Journal of Clinical Microbiology, Vol. 42, no. 10, 4419-4431, 2004.

[3] Stokes. Principles of Cytocentrifugation, Ph.D. Thesis Wescor, Logan, UT, USA, 2004. doi: 10.1309.

[4] E. Piaton, J. Faynel, K. Hutin, M.C. Ranchin, M. Cottier. Conventional liquid-based techniques versus Cytyc Thinprep ${ }^{\circledR}$ processing of urinary samples: a qualitative approach, BMC Clinical Pathology, Vol. 5, pp. 9, 2005. doi: 10 1186/1472-6890/5/9.

[5] L.D. Gray and G.D. Roberts. Laboratory diagnosis of systemic fungal diseases, Infectious Disease Clinics of North America, Vol. 2, pp. 779-803, 1988.

[6] P. Sandven and J. Lassen. Importance of selective media for recovery of yeasts from clinical specimens, Journal of Clinical Microbiology, Vol. 37, pp. 3731-3732, 1999.

[7] T. Scognamiglio, R. Zinchuk, P. Gumpeni, D.H. Larone. Comparison of IMA to SDA as the primary media for isolation of Fungi. Journal of Clinical Microbiology, Vol. 48, no. 5, pp. 1924-1925, 2010.

[8] D.H. Larone. Medically important Fungi: a guide to identification, 4th ed. American Society for Microbiology Press, Washington, DC, 2002.

[9] D.A. Sutton. Manual of Clinical Microbiology, 9th ed. American Society for Microbiology press, Washington, DC, 2007.

[10] C.W. Emmons, C.H. Binford and J.P. Uty. Medical mycology, 2nd ed., Philadelphia: Lea and Febiger, 1970.

[11] G.L. Woods, E. Pentony, M.J. Boxley, A.M. Gatson. Concentration of Sputum by Cytocentrifugation for Preparation of Smears for Detection of Acid-Fast Bacilli does Not Increase Sensitivity of the Fluorochrome Stain, Journal of Clinical Microbiology, Vol. 33, No. 7, pp. 1915-1916, 1995.

[12] C.A. Saceanu, N.C. Pfeiffer and T. Mclean. Evaluation of Sputum Smears Concentrated by Cytocentrifugation for Detection of Acid-Fast Bacilli, Journal of Clinical Microbiology, Vol. 31, No. 9, pp. 2317-2374, 1993.

[13] M. Singh, L. Khan, Y.N. Verma, N. Sachan, C. Pantola, A. Pathak, R. Gulati, A. Gautam. Comparatiive study for the use of different techniques in serous fluid cytology, Journal of Evolution of Medical and Dental Sciences, Vol. 4, No. 18, pp. 3154-3161, 2015. 